\title{
BRANNER-HUBBARD-LAVAURS DEFORMATIONS FOR REAL CUBIC POLYNOMIALS WITH A PARABOLIC FIXED POINT
}

\author{
SHIZUO NAKANE
}

\begin{abstract}
In this article, we study what we call the Branner-HubbardLavaurs deformation of real cubic polynomials with a parabolic fixed point of multiplier one. It turns out that the existence of non-trivial deformations corresponds to the oscillation of stretching rays and discontinuity of the wring operation.
\end{abstract}

\section{INTRODUCTION}

This article is a continuation of Komori-Nakane $\mathrm{KN}$ and considers the parameter space of a family of real cubic polynomials. A basic tool to investigate the parameter space is the wring deformation (or the Branner-Hubbard deformation). This deformation yields a stretching ray, which is a higher degree generalization of the external ray (parameter ray) of the Mandelbrot set. Although all external rays of the Mandelbrot set are conjectured to land, Komori and Nakane KN] has shown that, in the family of real cubic polynomials, certain stretching rays have non-trivial accumulation sets on the locus $\operatorname{Per}_{1}(1)$ where the maps have a parabolic fixed point of multiplier one; see Figure 1 .

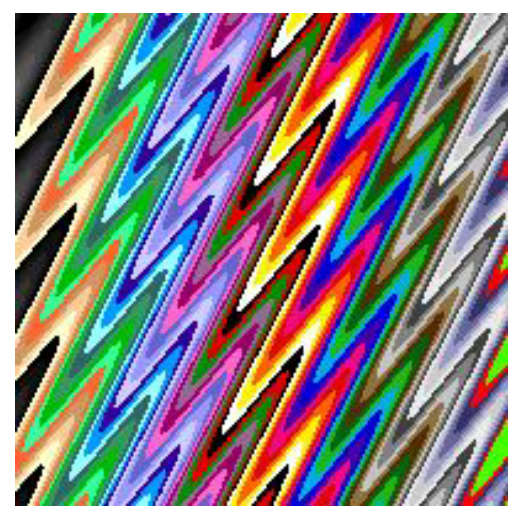

Figure 1. Oscillating rays

Received by the editors July 10, 2008.

2000 Mathematics Subject Classification. Primary 37F45; Secondary 37F30.

Key words and phrases. Branner-Hubbard deformation, Lavaurs map.

The author was supported in part by Grant-in-Aid for Scientific Research (No.17540177), Japan Society for the Promotion of Science.

(C)2009 American Mathematical Society Reverts to public domain 28 years from publication 
Since the wring deformation changes nothing on the filled-in Julia set, it does not deform polynomials with connected Julia sets. Whereas, for polynomials with parabolic cycles, the Lavaurs maps enable us to deform complex structures also in the parabolic basins. This deformation, the Branner-Hubbard-Lavaurs deformation, can deform such polynomials. Investigating the close connection between the wring deformation and the Branner-Hubbard-Lavaurs deformation around the parabolic locus $P e r_{1}(1)$, we will show that this actually happens on the locus $\operatorname{Per}_{1}(1)$; see Theorem 3.1. Of course, this is closely related to the non-landing of stretching rays and leads to the discontinuity of the wring operation on $\operatorname{Per}_{1}(1)$; see Corollary 3.1 .

Figure 1 also suggests that the oscillation of the stretching rays is quite regular. We will try to explain their regular oscillation in terms of the Branner-HubbardLavaurs deformation.

We also remark that Petersen and Tan Lei $[\mathrm{PT}]$ has investigated the BrannerHubbard-Lavaurs deformation of the quadratic map $z^{2}+1 / 4$ with a parabolic fixed point of multiplier one. Our work is a generalization of theirs to cubic polynomials.

The plan of this article is as follows. In Section 2, we give a short summary on the parameter space of real cubic polynomials and stretching rays. In Section 3, we define the Branner-Hubbard-Lavaurs deformation and state our main results. In Section 4, some facts on the accumulation sets of stretching rays obtained in [KN] are listed. In Section 5, we prove continuity of the accumulation sets of stretching rays. The proofs of theorems are given in Section 6.

\section{ReAl CUBiC POLYNOMials AND STRETCHING RAYS}

We consider a family of real cubic polynomials of the form:

$$
\mathcal{P}_{3}:=\left\{P(z)=P_{A, B}(z)=z^{3}-3 A z+\sqrt{B} ; A, B>0\right\},
$$

which can be regarded as $\mathbb{R}_{+}^{2}$. The filled-in Julia set $K(P)$ of such a polynomial $P$ is the set of points $z \in \mathbb{C}$ whose orbit $\left\{P^{n}(z) ; n \geq 0\right\}$ is bounded. Its boundary is called the Julia set of $P$ and is denoted by $J(P)$. The connectedness locus $\mathcal{C}_{3}$ of this family is the set of parameters $(A, B)$ such that $J\left(P_{A, B}\right)$ is connected. Its complement $\mathcal{E}_{3}=\mathbb{R}_{+}^{2} \backslash \mathcal{C}_{3}$ is the escape locus. Recall that $J(P)$ is connected if and only if both critical points $\pm \sqrt{A}$ of $P$ belong to $K(P)$. The shift locus $\mathcal{S}_{3}$ is the set of parameters both of whose critical points escape to $\infty$.

Milnor [M] gives a partition of our parameter space $\mathbb{R}_{+}^{2}$. Let us consider $P$ as a map from $\mathbb{R}$ to itself. Let $I$ be the smallest closed interval containing $K(P) \cap \mathbb{R}$. The set of $P$ so that $I$ consists of a single fixed point of $P$ denotes $\mathcal{R}_{0}$. For $1 \leq j \leq 3$, a map $P \notin \mathcal{R}_{0}$ belongs to $\mathcal{R}_{j}$ if the intersection of the graph of $P$ with $I \times I$ has $j$ distinct components; see Figure 2 for this partition and Figure 3 for the graphs of maps in each $\mathcal{R}_{j}, 0 \leq j \leq 3$.

Now the next proposition easily follows from the definition.

Proposition $2.1(\underline{\mathrm{M}})$ ). The connectedness locus $\mathcal{C}_{3}$ coincides with $\mathcal{R}_{1}$ and is bounded by the curves:

$$
\begin{aligned}
\operatorname{Per}_{1}(1) & : \quad B=(A+1 / 3)^{3}, \\
\operatorname{Preper}_{(1) 1} & : \quad B=4 A(A-1)^{2} .
\end{aligned}
$$

The union $\mathcal{R}_{0} \cup \mathcal{R}_{3}$ is contained in the shift locus $\mathcal{S}_{3}$.

The set $\mathcal{R}_{1}=\mathcal{C}_{3}$ is the gray region in Figure 2 , 


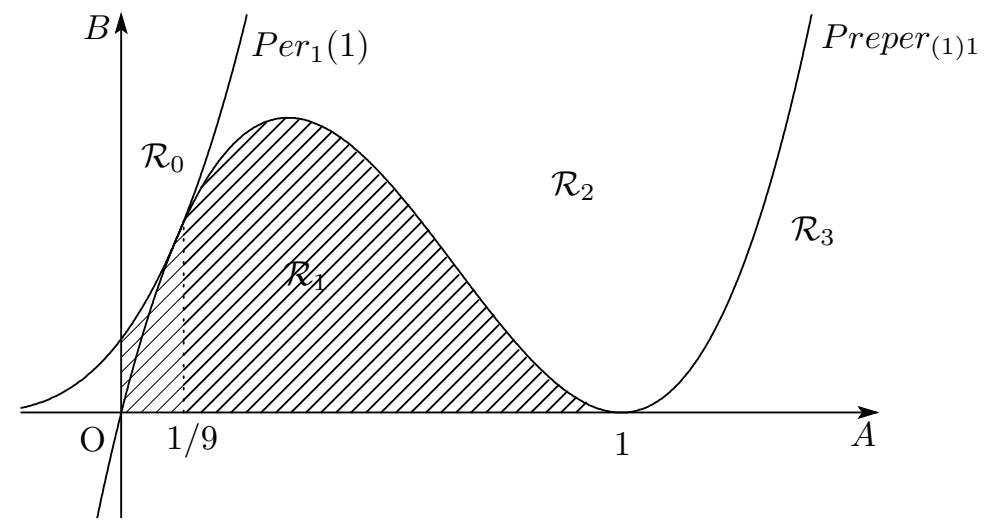

Figure 2. Parameter space of real cubic polynomials

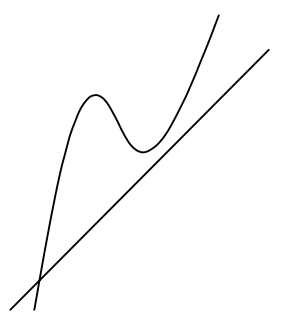

$\mathcal{R}_{0}$

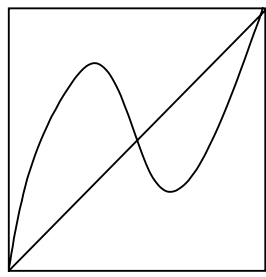

$\mathcal{R}_{1}$

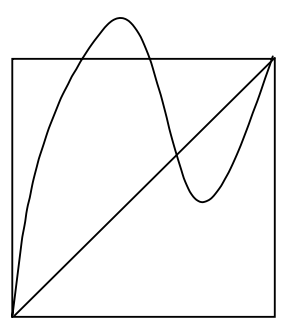

$\mathcal{R}_{2}$

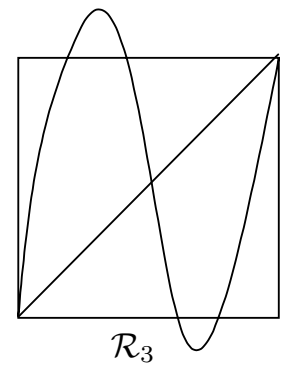

FiguRE 3. Graphs of maps in each $\mathcal{R}_{j}$

The locus $\operatorname{Per}_{1}(1)$ is the set of parameters which has a parabolic fixed point of multiplier one, while the locus Preper $_{(1) 1}$ is the set of parameters one of whose critical values is a fixed point. Our main interest lies in $\operatorname{Per}_{1}(1)$.

For $P \in \mathcal{P}_{3}$, the Green function $G_{P}$ of $K(P)$ is expressed by

$$
G_{P}(z)=\lim _{n \rightarrow \infty} 3^{-n} \log _{+}\left|P^{n}(z)\right|,
$$

where $\log _{+} t=\max \{\log t, 0\}$. It measures the escape rate of the point $z$ to $\infty$. It is continuous in $\mathbb{C}$, positive and harmonic in $\mathbb{C} \backslash K(P)$ and satisfies $G_{P}(z)=$ $\log |z|+O(1)$ as $z \rightarrow \infty$. It also satisfies

$$
G_{P}(P(z))=3 \cdot G_{P}(z) .
$$

We set

$$
H(P):=\max \left\{G_{P}(-\sqrt{A}), G_{P}(\sqrt{A})\right\}, \quad U_{P}:=\left\{z \in \mathbb{C} ; G_{P}(z)>H(P)\right\} .
$$

The Böttcher coordinate $\varphi_{P}$ of $P$ is a conformal map: $U_{P} \rightarrow\left\{z \in \mathbb{C} ;|z|>e^{H(P)}\right\}$ with the following property:

$$
\varphi_{P} \circ P(z)=\varphi_{P}(z)^{3}, \quad \lim _{z \rightarrow \infty} \frac{\varphi_{P}(z)}{z}=1 .
$$

Then we have $G_{P}(z)=\log \left|\varphi_{P}(z)\right|$ in $U_{P}$. 
For a positive number $s>0$, the map $\ell_{s}(z)=z|z|^{s-1}$ is quasiconformal (qc for short) and we define a $P$-invariant Beltrami form $\mu_{s}$ by

$$
\mu_{s}:=\left\{\begin{array}{l}
\left(\ell_{s} \circ \varphi_{P}\right)^{*} \mu_{0} \text { in } U_{P} \\
\mu_{0} \text { on } K(P) .
\end{array}\right.
$$

Here $\mu_{0}$ is the 0 -form. Then, by the Measurable Riemann Mapping Theorem, $\mu_{s}$ is integrated by a unique qc-map $\chi_{s}$ such that

$$
P_{s}:=\chi_{s} \circ P \circ \chi_{s}^{-1} \in \mathcal{P}_{3}, \quad \lim _{z \rightarrow \infty} \frac{\ell_{s} \circ \varphi_{P} \circ \chi_{s}^{-1}(z)}{z}=1 .
$$

Thus we define a real analytic map $W_{P}: \mathbb{R}_{+} \rightarrow \mathcal{P}_{3}$ by $W_{P}(s)=P_{s}$. The Böttcher coordinate $\varphi_{P_{s}}$ of $P_{s}$ is equal to $\ell_{s} \circ \varphi_{P} \circ \chi_{s}^{-1}$. Since $P_{s}$ is hybrid equivalent to $P$, it holds $P_{s} \equiv P$ for $P \in \mathcal{C}_{3}$. For $P \in \mathcal{E}_{3}$, we define the stretching ray ( $S$-ray for short) through $P$ by

$$
R(P):=W_{P}\left(\mathbb{R}_{+}\right)=\left\{P_{s} ; s \in \mathbb{R}_{+}\right\} .
$$

The Böttcher vector $\eta: \mathcal{S}_{3} \rightarrow \mathbb{R}$ is defined by

$$
\eta(P):=\frac{1}{\log 3}\left(\log G_{P}(-\sqrt{A})-\log G_{P}(\sqrt{A})\right) .
$$

From (1), we have, for any $n \in \mathbb{N}$,

$$
\eta(P)=\frac{1}{\log 3}\left(\log G_{P}\left(P^{n}(-\sqrt{A})\right)-\log G_{P}\left(P^{n}(\sqrt{A})\right)\right) .
$$

Then, it easily follows from Figure 3 that, in $\mathcal{S}_{3} \cap\left(\mathcal{R}_{0} \cup \mathcal{R}_{2}\right),-\sqrt{A}$ escapes to $\infty$ faster than $\sqrt{A}$, hence $\eta(P)>0$. It is true also in $\mathcal{R}_{3}$; see $\mathrm{KN}$, Lemma 3.3].

Lemma 2.1 ([KN, Prop. 2.1]). The Böttcher vector is constant on each S-ray in the shift locus $\mathcal{S}_{3}$.

Proof. If we take $n$ large so that $P^{n}( \pm \sqrt{A}) \in U_{P}$, we have

$$
\begin{aligned}
\log G_{P_{s}}\left(P_{s}^{n}\left( \pm \sqrt{A_{s}}\right)\right) & =\log \log \left|\varphi_{P_{s}}\left(P_{s}^{n}\left( \pm \sqrt{A_{s}}\right)\right)\right| \\
& =\log \log \left|\ell_{s} \circ \varphi_{P}\left(P^{n}( \pm \sqrt{A})\right)\right| \\
& =\log \log \left|\varphi_{P}\left(P^{n}( \pm \sqrt{A})\right)\right|+\log s \\
& =\log G_{P}\left(P^{n}( \pm \sqrt{A})\right)+\log s .
\end{aligned}
$$

Thus it follows that $\eta\left(P_{s}\right) \equiv \eta(P)$. This completes the proof.

Thus, each S-ray in $\mathcal{S}_{3}$ forms a level curve of the Böttcher vector map $P \mapsto \eta(P)$. S-rays in $\mathcal{S}_{3}$ can be easily drawn by the Böttcher vector; see Figure 1 .

Note that the above construction works also for general complex cubic polynomials and for non-real $s$ in the right half plane $\mathbb{H}:=\{s=t+i u \in \mathbb{C} ; t>0\}$. Then the deformation $P_{s}$ is called the wring deformation or the Branner-Hubbard deformation of $P$. Lemma 2.1 is true also in the shift locus of the complex cubic polynomials. Basic references are [B], $[\mathrm{BH}], \mathrm{T}]$ and $[\mathrm{W}]$. 


\section{BRANNER-HuBBARD-LAVAURS DEFORMATIONS FOR REAL CUBIC POLYNOMIALS WITH A PARABOLIC FIXED POINT}

Consider the locus $\operatorname{Per}_{1}^{*}(1):=\left\{B=4(A+1 / 3)^{3} ; 0<A<1 / 9\right\} \subset \operatorname{Per}_{1}(1)$, where the corresponding map $Q=Q_{A}:=P_{A, 4(A+1 / 3)^{3}}$ has a parabolic fixed point $\beta_{Q}=\sqrt{A+1 / 3}$ of multiplier one whose immediate basin $\mathcal{B}_{Q}$ contains both critical points. Let $\phi_{Q,-}$ and $\phi_{Q,+}$ denote the attracting and repelling Fatou coordinates respectively of $\beta_{Q}$ for $Q \in \operatorname{Per}_{1}^{*}(1)$. Since the Fatou coordinates have ambiguity of additive constants, we normalize them so that they are symmetric with respect to the real axis. Especially, we normalize the attracting Fatou coordinate by $\phi_{Q,-}(\sqrt{A})=0$. Put $\psi_{Q,+}=\phi_{Q,+}^{-1}$. The Lavaurs map $g_{Q, \sigma}: \mathcal{B}_{Q} \rightarrow \mathbb{C}$ with lifted phase $\sigma \in \mathbb{R}$ is defined by $g_{Q, \sigma}=\psi_{Q,+} \circ T_{\sigma} \circ \phi_{Q,-}$, where $T_{\sigma}(z)=z+\sigma$. Since everything is symmetric with respect to the real axis, we have $g_{Q, \sigma}( \pm \sqrt{A}) \in \mathbb{R} \backslash K(Q)$, hence $\varphi_{Q}\left(g_{Q, \sigma}( \pm \sqrt{A})\right)>1$. Then it is easy to see $g_{Q, \sigma}(\sqrt{A})=\psi_{Q,+}(\sigma)$, which we will often use later.

The basic underlying space we consider in what follows is $W=\operatorname{Per}_{1}^{*}(1) \times \mathbb{R}$, whose element is denoted by $w=(Q, \sigma)$. For $w \in W$, we define a $\left\langle Q, g_{w}\right\rangle$-invariant Beltrami form $\mu_{s, w}$ by

$$
\mu_{s, w}:=\left\{\begin{array}{l}
\left(\ell_{s} \circ \varphi_{Q}\right)^{*} \mu_{0} \text { in } \mathbb{C} \backslash K(Q), \\
\left(g_{w}^{n} \circ Q^{m}\right)^{*} \mu_{s, w} \text { in } \mathcal{B}_{Q} \cap Q^{-m} g_{w}^{-n}(\mathbb{C} \backslash K(Q)), \\
\mu_{0} \text { otherwise. }
\end{array}\right.
$$

Then, as before, there exists a unique integrating qc-map $\chi_{s, w}$ of $\mu_{s, w}$ such that

$$
Q_{s, w}:=\chi_{s, w} \circ Q \circ \chi_{s, w}^{-1} \in \operatorname{Per}_{1}^{*}(1), \quad \lim _{z \rightarrow \infty} \frac{\ell_{s} \circ \varphi_{Q} \circ \chi_{s, w}^{-1}(z)}{z}=1 .
$$

Since $g_{Q, \sigma} \circ Q=g_{Q, \sigma+1}$, we have $\mu_{s, Q, \sigma+1}=\mu_{s, Q, \sigma}$. Then, by uniqueness, it follows $\chi_{s, Q, \sigma+1}=\chi_{s, Q, \sigma}$ and $Q_{s, Q, \sigma+1}=Q_{s, Q, \sigma}$. If $s=1$, we have $\mu_{1, w}=\mu_{0}, \chi_{1, w}=i d$, hence $Q_{1, w}=Q$. Note that $\varphi_{Q_{s, w}}=\ell_{s} \circ \varphi_{Q} \circ \chi_{s, w}^{-1}$.

Lemma 3.1 ([KN, p. 112] and [ $\mathrm{PT}$, Theorem 4.6]). $\chi_{s, w} \circ g_{w} \circ \chi_{s, w}^{-1}$ is also a Lavaurs map of $Q_{s, w}$ with some lifted phase $\Sigma(s, w): \chi_{s, w} \circ g_{w} \circ \chi_{s, w}^{-1}=g_{Q_{s, w}, \Sigma(s, w)}$.

Then $\Sigma$ is a map from $\mathbb{R}_{+} \times W$ to $\mathbb{R}$.

Lemma 3.2. We have $\psi_{Q_{s, w},+}(\Sigma(s, w))=\chi_{s, w} \circ \psi_{Q,+}(\sigma)$. Hence, the map $s \mapsto$ $\Sigma(s, w)$ is real analytic. Moreover, $\Sigma(s, Q, \sigma+k)=\Sigma(s, Q, \sigma)+k$ for any $k \in \mathbb{N}$.

Proof. The first statement of this lemma follows from the fact

$$
\begin{aligned}
\psi_{Q_{s, w},+}(\Sigma(s, w)) & =g_{Q_{s, w}, \Sigma(s, w)}\left(\sqrt{A_{s, w}}\right) \\
& =\chi_{s, w} \circ g_{w} \circ \chi_{s, w}^{-1}\left(\sqrt{A_{s, w}}\right) \\
& =\chi_{s, w} \circ \psi_{Q,+} \circ T_{\sigma} \circ \phi_{Q,-}(\sqrt{A}) \\
& =\chi_{s, w} \circ \psi_{Q,+}(\sigma)
\end{aligned}
$$

and the local injectivity of $\psi_{Q_{s, w},+}$. The second one follows from:

$$
\begin{aligned}
\chi_{s, w} \circ g_{Q, \sigma+k} \circ \chi_{s, w}^{-1} & =\chi_{s, w} \circ g_{w} \circ Q^{k} \circ \chi_{s, w}^{-1} \\
& =g_{Q_{s, w}, \Sigma(s, w)} \circ Q_{s, w}^{k} \\
& =g_{Q_{s, w}, \Sigma(s, w)+k}
\end{aligned}
$$


Lemma 3.3. For any $w \in W, \Sigma(s, w)$ tends to $+\infty$ or to $-\infty$ as $s$ tends to $+\infty$ or to 0 respectively.

Proof. From Lemma 3.2, we have

$$
\begin{aligned}
\varphi_{Q_{s, w}} \circ \psi_{Q_{s, w},+}(\Sigma(s, w)) & =\ell_{s} \circ \varphi_{Q} \circ \chi_{s, w}^{-1} \circ \chi_{s, w} \circ \psi_{Q,+}(\sigma) \\
& =\ell_{s} \circ \varphi_{Q} \circ \psi_{Q,+}(\sigma),
\end{aligned}
$$

which tends to $+\infty$ as $s \rightarrow+\infty$ and to 1 as $s \rightarrow 0$. Since $Q_{s, w}$ belongs to a compact subset $\overline{\operatorname{Per}_{1}^{*}(1)}$ in $\operatorname{Per}_{1}(1), \psi_{Q_{s, w},+}(\Sigma(s, w))$ tends to $+\infty$ as $s \rightarrow+\infty$ and is arbitrarily close to $\beta_{Q_{s, w}}$ as $s \rightarrow 0$. Hence $\Sigma(s, w)$ tends to $+\infty$ or to $-\infty$ as $s$ tends to $+\infty$ or to 0 respectively.

We call $L_{s}(w):=\left(Q_{s, w}, \Sigma(s, w)\right) \in W$ the Branner-Hubbard-Lavaurs deformation (BHL-deformation for short) of $w$. Note that $L_{1}(w)=w$. We define the $B H L$-ray $L(w)$ through $w$ by

$$
L(w):=\left\{L_{s}(w) \in W ; s \in \mathbb{R}_{+}\right\},
$$

which is actually a ray in $W$ by Lemma 3.3. Recall that $\varphi_{Q}\left(g_{w}( \pm \sqrt{A})\right)>1$. The Böttcher-Lavaurs vector $\eta: W \rightarrow \mathbb{R}$ is defined by

$$
\eta(w):=\frac{1}{\log 3}\left(\log \log \varphi_{Q}\left(g_{w}(-\sqrt{A})\right)-\log \log \varphi_{Q}\left(g_{w}(\sqrt{A})\right)\right) .
$$

It satisfies $\eta(Q, \sigma+1)=\eta(Q, \sigma)$. Thus $\eta(w)$ can be regarded as a function also on $\operatorname{Per}_{1}^{*}(1) \times \mathbb{R} / \mathbb{Z}$.

By the same argument as in the proof of Lemma 2.1, we have the following.

Lemma 3.4. The Böttcher-Lavaurs vector $\eta(w)$ is constant on each BHL-ray.

Proof. From the definition, it follows that

$$
\begin{aligned}
\log \log \varphi_{Q_{s, w}}\left(g_{Q_{s, w}, \Sigma(s, w)}\left( \pm \sqrt{A_{s, w}}\right)\right) & =\log \log \ell_{s} \circ \varphi_{Q}\left(g_{w}( \pm \sqrt{A})\right) \\
& =\log \log \varphi_{Q}\left(g_{w}( \pm \sqrt{A})\right)+\log s .
\end{aligned}
$$

Thus $\eta\left(Q_{s, w}, \Sigma(s, w)\right) \equiv \eta(w)$.

We define the Fatou vector $\tau: \operatorname{Per}_{1}^{*}(1) \rightarrow \mathbb{R}_{+}$by $\tau(Q):=\phi_{Q,-}(-\sqrt{A})-$ $\phi_{Q,-}(\sqrt{A})$.

Lemma 3.5 ([KN, Prop. 6.5]). The Fatou vector gives a parametrization of the locus $\operatorname{Per}_{1}^{*}(1)$.

For each $k \in \mathbb{N}$, there exists a unique map $Q_{k} \in \operatorname{Per}_{1}^{*}(1)$ satisfying $\tau\left(Q_{k}\right)=k$. It satisfies the critical orbit relation $Q_{k}(-\sqrt{A})=Q_{k}^{k+1}(\sqrt{A})$, which is preserved under BHL-deformation. Hence it follows that $Q_{s, w} \equiv Q$ and $\eta(Q, \sigma) \equiv k$ for $Q=Q_{k}, k \in \mathbb{N}$. The following is the main theorem of this article.

Theorem 3.1 (Non-trivial BHL-deformation). For any $w=(Q, \sigma) \in W$ with $\tau(Q) \notin \mathbb{N}$, the map $s \mapsto Q_{s, w}$ is not constant.

Such a map was first obtained in Willumsen $[\mathrm{W}$ in the region $A<0$; see also Tan Lei $\mathrm{T}$. Once we get such a non-trivial deformation, the following corollary is essentially due to [W] (see Lemma 6.7).

Corollary 3.1 (Discontinuity of wring operation). Suppose $\tau(Q) \notin \mathbb{N}$. Then the map $(P, s) \mapsto W_{P}(s)$ is discontinuous at $(Q, s)$ if $Q_{s, w} \neq Q$ for some $\sigma$. 


\section{Accumulation of Stretching Rays in $\mathcal{R}_{0}$}

Recall that the region $\mathcal{R}_{0}=\left\{B>4(A+1 / 3)^{3}\right\}$ is contained in the shift locus. S-rays in $\mathcal{R}_{0}$ are uniquely labelled by the Böttcher vector; see Section 6.3 in [KN]. Let $R(\eta)$ denote the S-ray in $\mathcal{R}_{0}$ with Böttcher vector $\eta>0$ and let $I(\eta)$ be the accumulation set of $R(\eta)$, which is a compact connected subset of $\operatorname{Per}_{1}^{*}(1)$. The following is the main theorem in $[\mathrm{KN}]$ and is the key to the proof of Theorem 3.1.

Proposition 4.1 ([ $[\mathrm{KN}$, Non-landing of S-rays $]$ ). If $k \in \mathbb{N}$, then $I(k)=\left\{Q_{k}\right\}$, that is, $R(k)$ lands at $Q_{k}$. If $\eta \notin \mathbb{N}$, then $I(\eta)$ is a non-trivial arc on $\operatorname{Per}_{1}^{*}(1)$.

See Figure 1 for non-landing S-rays. The following gives a characterization of the set $I(\eta)$ and will be often used later.

Lemma 4.1 ([KN, Corollary 7.2]). The set $I(\eta)$ is equal to the set of $Q \in \operatorname{Per}_{1}^{*}(1)$ such that $\eta(Q, \sigma)=\eta$ for some $\sigma$. That is, $I(\eta)$ coincides with the projection to the $Q$-axis of the level set $\eta(Q, \sigma)=\eta$.

The next theorem suggests that S-rays are obtained from the rescaling of BHLrays and seems to explain the regular oscillation of S-rays.

Theorem 4.1. The projection to the $Q$-axis of the BHL-ray $L(w)$ through $w \in W$ coincides with $I(\eta(w))$.

\section{Continuity of the aCCUMUlation Sets}

The main purpose of this section is to prove the following proposition, which will be used in the next section.

Proposition 5.1. The set $I(\eta)$ depends continuously on $\eta$.

To prove this, we need some lemmas. Put $\eta_{M}(Q)=\max \{\eta(Q, \sigma) ; \sigma \in \mathbb{R} / \mathbb{Z}\}$ and $\eta_{m}(Q)=\min \{\eta(Q, \sigma) ; \sigma \in \mathbb{R} / \mathbb{Z}\}$. Since $\eta\left(Q_{k}, \sigma\right)=k$ for any $\sigma$, we have $\eta_{M}\left(Q_{k}\right)=\eta_{m}\left(Q_{k}\right)=k$.

Lemma 5.1. The functions $\eta_{M}$ and $\eta_{m}$ do not depend on the choice of Fatou coordinates.

Proof. If we take another pair of Fatou coordinates $\tilde{\phi}_{Q, \pm}(z)=\phi_{Q, \pm}(z)+c_{ \pm}, c_{ \pm} \in \mathbb{R}$, the corresponding Lavaurs maps are written by

$$
\tilde{g}_{Q, \sigma}:=\tilde{\psi}_{Q,+} \circ T_{\sigma} \circ \tilde{\phi}_{Q,-}=\psi_{Q,+} \circ T_{\sigma-c_{+}+c_{-}} \circ \phi_{Q,-}=g_{Q, \sigma-c_{+}+c_{-}} .
$$

Hence the corresponding Böttcher-Lavaurs vector is written by $\tilde{\eta}(Q, \sigma)=$ $\eta\left(Q, \sigma-c_{+}+c_{-}\right)$. Now the lemma easily follows.

Lemma 5.2. The functions $\eta_{M}$ and $\eta_{m}$ are continuous in $\operatorname{Per}_{1}^{*}(1)$.

Proof. Put $\eta_{M}(Q)=\eta\left(Q, \sigma_{Q}\right)$ for each $Q \in \operatorname{Per}_{1}^{*}(1)$. From the uniform continuity of $\eta(Q, \sigma)$, it follows that for any $\epsilon>0$ there exists $\delta>0$ such that, if $\left|Q-Q^{(0)}\right|<\delta$, then $\left|\eta(Q, \sigma)-\eta\left(Q^{(0)}, \sigma\right)\right|<\epsilon$ for any $\sigma \in \mathbb{R} / \mathbb{Z}$. Thus, if $\left|Q-Q^{(0)}\right|<\delta$, we have

$$
\begin{aligned}
& \eta_{M}(Q)=\eta\left(Q, \sigma_{Q}\right) \leq \eta\left(Q^{(0)}, \sigma_{Q}\right)+\epsilon \leq \eta_{M}\left(Q^{(0)}\right)+\epsilon, \\
& \eta_{M}(Q) \geq \eta\left(Q, \sigma_{Q^{(0)}}\right) \geq \eta\left(Q^{(0)}, \sigma_{Q^{(0)}}\right)-\epsilon=\eta_{M}\left(Q^{(0)}\right)-\epsilon .
\end{aligned}
$$

This implies the continuity of $\eta_{M}$. The case of $\eta_{m}$ is similar.

Lemma 5.3. The functions $\eta_{M}$ and $\eta_{m}$ are strictly increasing in $\operatorname{Per}_{1}^{*}(1)$. 
Proof. We have only to show the claim in each interval $Q_{k} \leq Q \leq Q_{k+1}$ of $\operatorname{Per}_{1}^{*}(1)$. Since $I(\eta)$ is compact and connected, it is a closed interval of $\operatorname{Per}_{1}^{*}(1)$. Put $A_{c}:=$ $\left\{(\eta, Q) \in \mathbb{R} \times \operatorname{Per}_{1}^{*}(1) ; Q \in I(\eta)\right\}$. By Lemma 4.1 we have $A_{c}=\{(\eta, Q) \in$ $\left.\mathbb{R} \times \operatorname{Per}_{1}^{*}(1) ; \eta_{m}(Q) \leq \eta \leq \eta_{M}(Q)\right\}$; see Figure 4. Suppose $\eta_{M}$ is not monotone increasing. Then it has a local maximum $\eta_{0}$ at some point $Q^{(0)}$. Hence the set $I\left(\eta_{0}\right)=\left\{Q \in \operatorname{Per}_{1}^{*}(1) ; \eta_{m}(Q) \leq \eta_{0} \leq \eta_{M}(Q)\right\}$ consists of $\left\{Q^{(0)}\right\}$ and another connected component; that is, it cannot be connected, a contradiction.

To show that $\eta_{M}$ is strictly increasing, suppose $\eta_{M}(Q) \equiv \eta_{0}$ in a non-trivial closed interval $\left[Q^{\prime}, Q^{\prime \prime}\right] \subset\left[Q_{k}, Q_{k+1}\right]$. Take $Q^{(0)} \in\left(Q^{\prime}, Q^{\prime \prime}\right)$ and put $\eta\left(Q^{(0)}, \sigma_{0}\right)=$ $\eta_{M}\left(Q^{(0)}\right)=\eta_{0}$. By Proposition 7.3 in [KN], there exists a choice of Fatou coordinates $\tilde{\phi}_{Q, \pm}$ such that the corresponding function $\tilde{\eta}(Q, \sigma)$ satisfies $\tilde{\eta}\left(Q^{(0)}, \sigma_{0}\right)=\eta_{0}$ and $Q \mapsto \tilde{\eta}\left(Q, \sigma_{0}\right)$ is strictly increasing. Then $\tilde{\eta}\left(Q, \sigma_{0}\right)>\tilde{\eta}\left(Q^{(0)}, \sigma_{0}\right)=\eta_{0}$ for $Q>Q^{(0)}$, hence $\tilde{\eta}_{M}(Q)>\eta_{0}$ for $Q>Q^{(0)}$. This contradicts Lemma 5.1 This completes the proof.

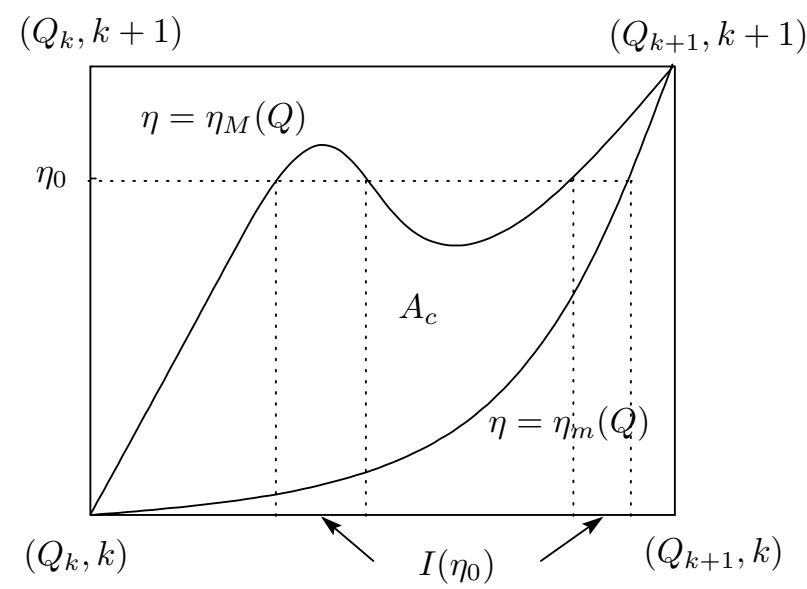

FIGURE 4. $I\left(\eta_{0}\right)$ is disconnected if $\eta_{M}$ is not monotone

Proof of Proposition 5.1. Thus the functions $\eta=\eta_{M}(Q)$ and $\eta=\eta_{m}(Q)$ have continuous inverses $Q=Q_{M}(\eta)$ and $Q=Q_{m}(\eta)$ respectively. Then $I(\eta)=\{Q \in$ $\left.\operatorname{Per}_{1}^{*}(1) ; Q_{M}(\eta) \leq Q \leq Q_{m}(\eta)\right\}$. This completes the proof of Proposition 5.1.

\section{Proof of theorems}

In this section, we will prove Theorems 3.1, 4.1 and Corollary 3.1

Lemma 6.1. For $s, s^{\prime}>0$, we have $L_{s^{\prime}} \circ L_{s}=L_{s s^{\prime}}$.

Proof. Note that $\ell_{s^{\prime}} \circ \ell_{s}=\ell_{s s^{\prime}}$. Let $\mu_{s, s^{\prime}}$ be the $\left\langle Q_{s, w}, g_{Q_{s, w}, \Sigma(s, w)}\right\rangle$-invariant Beltrami form:

$$
\mu_{s, s^{\prime}}:=\left\{\begin{array}{l}
\left(\ell_{s^{\prime}} \circ \varphi_{Q_{s, w}}\right)^{*} \mu_{0} \text { in } \mathbb{C} \backslash K\left(Q_{s, w}\right), \\
\left(g_{Q_{s, w}, \Sigma(s, w)}^{n} \circ Q_{s, w}^{m}\right)^{*} \mu_{s, s^{\prime}} \text { in } \mathcal{B}_{Q_{s, w}} \cap Q_{s, w}^{-m} g_{Q_{s, w}, \Sigma(s, w)}^{-n}\left(\mathbb{C} \backslash K\left(Q_{s, w}\right)\right), \\
\mu_{0} \text { otherwise }
\end{array}\right.
$$


and let $\chi_{s, s^{\prime}}$ be the unique integrating qc-map of $\mu_{s, s^{\prime}}$ satisfying $Q_{s, s^{\prime}}:=\chi_{s, s^{\prime}} \circ$ $Q_{s, w} \circ \chi_{s, s^{\prime}}^{-1} \in \operatorname{Per}_{1}^{*}(1)$. Then $L_{s^{\prime}}\left(Q_{s, w}, \Sigma(s, w)\right)=\left(Q_{s, s^{\prime}}, \Sigma\left(s^{\prime}, Q_{s, w}, \Sigma(s, w)\right)\right)$ and we have in $\mathbb{C} \backslash K(Q)$,

$$
\begin{aligned}
\chi_{s, w}^{*} \mu_{s, s^{\prime}} & =\chi_{s, w}^{*}\left(\ell_{s^{\prime}} \circ \varphi_{Q_{s, w}}\right)^{*} \mu_{0} \\
& =\chi_{s, w}^{*}\left(\ell_{s^{\prime}} \circ \ell_{s} \circ \varphi_{Q} \circ \chi_{s, w}^{-1}\right)^{*} \mu_{0} \\
& =\left(\ell_{s s^{\prime}} \circ \varphi_{Q}\right)^{*} \mu_{0} \\
& =\mu_{s s^{\prime}, w} .
\end{aligned}
$$

By the $\left\langle Q, g_{s s^{\prime}, w}\right\rangle$-invariance of $\mu_{s s^{\prime}, w}$ and $\left\langle Q_{s, w}, g_{Q_{s, w}, \Sigma(s, w)}\right\rangle$-invariance of $\mu_{s, s^{\prime}}$, this equality is inherited to $K(Q)$. Hence we have $\left(\chi_{s, s^{\prime}} \circ \chi_{s, w}\right)^{*} \mu_{0}=\chi_{s, w}^{*} \mu_{s, s^{\prime}}=$ $\mu_{s s^{\prime}, w}$ in $\mathbb{C}$ and $\chi_{s, s^{\prime}} \circ \chi_{s, w} \circ \chi_{s s^{\prime}, w}^{-1}$ gives a conformal equivalence $Q_{s s^{\prime}, w} \sim Q_{s, s^{\prime}}$. This implies $Q_{s s^{\prime}, w}=Q_{s, s^{\prime}}$ and $\chi_{s s^{\prime}, w}=\chi_{s, s^{\prime}} \circ \chi_{s, w}$; see the following commutative diagram:

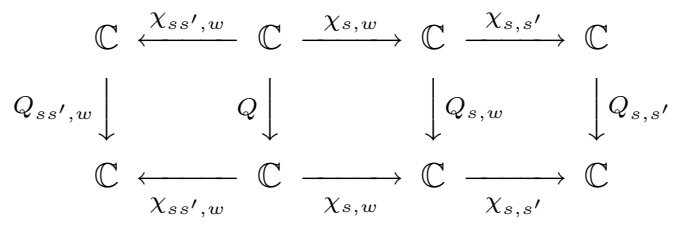

Next, by Lemma 3.2

$$
\begin{aligned}
\Sigma\left(s^{\prime}, \Sigma(s, w)\right) & =\phi_{Q_{s s^{\prime}, w},+} \circ g_{Q_{s s^{\prime}, \Sigma\left(s^{\prime}, Q_{w}, \Sigma(s, w)\right.}}\left(\sqrt{A_{s s^{\prime}, w}}\right) \\
& =\phi_{Q_{s s^{\prime}, w},+} \circ \chi_{s, s^{\prime}} \circ g_{Q_{s, w}, \Sigma(s, w)}\left(\sqrt{A_{s, w}}\right) \\
& =\phi_{Q_{s s^{\prime}, w},+} \circ \chi_{s, s^{\prime}} \circ \chi_{s, w} \circ g_{Q, w}(\sqrt{A}) \\
& =\phi_{Q_{s s^{\prime}, w},+} \circ \chi_{s s^{\prime}, w} \circ \psi_{Q,+}(\sigma) \\
& =\Sigma\left(s s^{\prime}, w\right) .
\end{aligned}
$$

This completes the proof.

Corollary 6.1. The map $s \mapsto L_{s}(w)$ is injective. Hence each BHL-ray has no self-intersection points.

Proof. Suppose it is not injective. We may assume there exist $s>1$ and $s^{\prime}>0$ such that $L_{s^{\prime}}(w)=L_{s s^{\prime}}(w)$. Then, by Lemma 6.1 we have $L_{s^{\prime}}(w)=L_{s^{k} s^{\prime}}(w)$, especially $\Sigma\left(s^{k} s^{\prime}, w\right)=\Sigma\left(s^{\prime}, w\right)$ for any $k \in \mathbb{N}$. This contradicts Lemma 3.3

Corollary 6.2. Two distinct BHL-rays never intersect.

Proof. Lemma 6.1 says that each BHL-ray is constructed by the BHL-deformation of arbitrary point on it. Thus if two BHL-rays have common points, they must coincide. This completes the proof.

Since the line $Q=Q_{k}$ in $W$ for $k \in \mathbb{N}$ forms a BHL-ray, the ray $L(w)$ with $Q_{k}<Q<Q_{k+1}$ stays in the strip $Q_{k}<Q<Q_{k+1}$ in $W$.

By Lemma 3.2 we can consider the BHL-deformation on the cylinder $\operatorname{Per}_{1}^{*}(1) \times$ $\mathbb{R} / \mathbb{Z}$, which we denote also by $L_{s}$.

Lemma 6.2. Each BHL-ray, projected on $\operatorname{Per}_{1}^{*}(1) \times \mathbb{R} / \mathbb{Z}$, is a closed loop. 
Proof. Suppose a BHL-ray $L(w)$ with $\eta(w)=\eta_{0}$ in the strip $Q_{k}<Q<Q_{k+1}$ is not closed on $\operatorname{Per}_{1}^{*}(1) \times \mathbb{R} / \mathbb{Z}$. It must wind countably many times around the cylinder. Then, for any fixed $\sigma_{0}$, the real analytic function $Q \mapsto \eta\left(Q, \sigma_{0}\right)$ has countably many $\eta_{0}$-points on the interval $Q_{k}<Q<Q_{k+1}$, which implies the function $\eta\left(Q, \sigma_{0}\right)$ is constant. Since all BHL-rays pass the line $\sigma=\sigma_{0}$, the map $\eta(Q, \sigma)$ must be constant. This is a contradiction. This completes the proof.

Thus, for any $(Q, \sigma) \in \operatorname{Per}_{1}^{*}(1) \times \mathbb{R}$, there exist $s_{0}>1$ and $k \in \mathbb{Z}$ such that $L_{s_{0}}(Q, \sigma)=(Q, \sigma+k)$. By Lemmas 6.1 and 3.2, this holds also for any point on $L(Q, \sigma)$. Moreover, by Lemma 6.1, we have $L_{s_{0}^{n}}(Q, \sigma)=(Q, \sigma+n k)$ for any $n \in \mathbb{N}$. Taking $n \rightarrow \infty$, we have $k>0$ by Lemma 3.3 .

Lemma 6.3. If we take a minimal $s_{0}>1$ such that $L_{s_{0}}(Q, \sigma)=(Q, \sigma+k)$ for some $k \in \mathbb{N}$, then $k=1$.

Proof. By the minimality of $s_{0}$, the curve $\left\{L_{s}(Q, \sigma) ; 1 \leq s \leq s_{0}\right\}$ in $\operatorname{Per}_{1}^{*}(1) \times \mathbb{R} / \mathbb{Z}$ is a simple loop and winds the cylinder $k$-times. Then there exist numbers $1<$ $s_{1}<s_{2}<\cdots<s_{k-1}<s_{0}$ and maps $Q^{(j)} \in \operatorname{Per}_{1}^{*}(1), 1 \leq j \leq k-1$ such that $L_{s_{j}}(Q, \sigma)=\left(Q^{(j)}, \sigma+j\right), 1 \leq j \leq k-1$. We may assume $Q<Q^{(1)}$. Since the loop is simple, we have $Q<Q^{(1)}<\cdots<Q^{(k-1)}$. Then it follows $Q^{(k-1)}<Q$ because $L_{s_{0}}(Q, \sigma)=(Q, \sigma+k)$, which is a contradiction.

We will show $s_{0}=3$.

Lemma 6.4. For any $(Q, \sigma) \in \operatorname{Per}_{1}^{*}(1) \times \mathbb{R}, L_{3}(Q, \sigma)=(Q, \sigma+1)$.

Proof. Let $s_{0}>1$ be the number satisfying $L_{s_{0}}(Q, \sigma)=(Q, \sigma+1)$. Recall that

$$
\varphi_{Q_{s_{0}, w}} \circ \psi_{Q_{s_{0}, w},+}\left(\Sigma\left(s_{0}, w\right)\right)=\ell_{s_{0}} \circ \varphi_{Q} \circ \psi_{Q,+}(\sigma) \text {. }
$$

From the assumption, the left hand side is equal to

$$
\begin{aligned}
\varphi_{Q} \circ \psi_{Q,+}(\sigma+1) & =\varphi_{Q} \circ \psi_{Q,+} \circ T_{1}(\sigma) \\
& =\ell_{3} \circ \varphi_{Q} \circ \psi_{Q,+}(\sigma) .
\end{aligned}
$$

Thus $s_{0}=3$.

Lemma 6.5. For any $w \in \operatorname{Per}_{1}^{*}(1) \times \mathbb{R}$ and $s>0$, we have $Q_{3 s, w}=Q_{s, w}$ and $\Sigma(3 s, w)=\Sigma(s, w)+1$.

Proof. It follows from Lemmas 6.1, 6.4 and 3.2 that

$$
\begin{aligned}
\left(Q_{3 s, w}, \Sigma(3 s, w)\right) & =L_{3 s}(w) \\
& =L_{s} \circ L_{3}(w) \\
& =L_{s}(Q, \sigma+1) \\
& =\left(Q_{s, Q, \sigma+1}, \Sigma(s, Q, \sigma+1)\right) \\
& =\left(Q_{s, w}, \Sigma(s, w)+1\right) .
\end{aligned}
$$

This completes the proof.

Lemma 6.6. Each BHL-ray forms a connected component of a level set of $\eta$.

Proof. Take a BHL-ray $\gamma_{0}$ and a point $p$ outside $\gamma_{0}$ but of the same level. Let $\gamma$ be the BHL-ray passing through $p$. Lemma 6.2 says $\gamma_{0}$ and $\gamma$ are loops on the cylinder. In the annulus bounded by these two loops, there exists a point $p^{\prime}$ of a different level, because $\eta$ is not constant. The BHL-ray passing through $p^{\prime}$ separates the 
rays $\gamma_{0}$ and $\gamma$. Thus $\gamma_{0}$ and $p$ do not belong to a same connected component of the level set.

Proof of Theorem 3.1. Suppose $Q_{s, w} \equiv Q^{(0)}$. By Lemma 3.3. the line $Q=Q^{(0)}$ in $W$ forms a BHL-ray, hence is contained in the level set of some level $\eta_{0}$. Suppose the level set of level $\eta_{0}$ has another connected component, say $\gamma$. Then its projection to the $Q$-axis consists of $\left\{Q^{(0)}\right\}$ and the projection of $\gamma$. This implies $I\left(\eta_{0}\right)$ is disconnected, which contradicts Lemma 4.1. Thus, the line $Q=Q^{(0)}$ is the unique component of the level set of $\eta$ of level $\eta_{0}$. Again by Lemma 4.1, we have $I\left(\eta_{0}\right)=$ $\left\{Q^{(0)}\right\}$, that is, $R\left(\eta_{0}\right)$ lands at $Q^{(0)}$. By Proposition 4.1, we conclude $\tau\left(Q^{(0)}\right) \in \mathbb{N}$. This completes the proof.

To prove Corollary [3.1, we need the following. Recall that Fatou coordinates are also defined for the perturbation $P \in \mathcal{R}_{0}$ off the locus $\operatorname{Per}_{1}(1)$. Let $\phi_{P,-}$ and $\phi_{P,+}$ denote respectively the incoming and outgoing Fatou coordinates of $P$. By an appropriate normalization, they are continuous up to $\operatorname{Per}_{1}(1)$. A relevant fact for perturbation is that the outgoing Fatou coordinate can be regarded also as an incoming one. Hence the difference $\sigma(P):=\phi_{P,+}(z)-\phi_{P,-}(z)$ is a constant, which we call the lifted phase of $P$. Roughly speaking, $-\sigma(P)$ is the time needed for the orbits of $P$ to pass through the gate between the bifurcating fixed points of $P$. Thus $\sigma(P) \rightarrow-\infty$ as $P$ tends to $\operatorname{Per}_{1}(1)$.

Lemma 6.7 ([W] Prop. 8.2] and [T, Key Continuity Theorem]). Suppose, as $n \rightarrow$ $\infty$,

(1) $P_{n} \rightarrow Q \in \operatorname{Per}_{1}^{*}(1), P_{n}$ has no attracting fixed points,

(2) $\sigma\left(P_{n}\right) \rightarrow \sigma$ in $\mathbb{C} / \mathbb{Z}$

(3) $K\left(P_{n}\right) \rightarrow K(Q, \sigma), J\left(P_{n}\right) \rightarrow J(Q, \sigma)$ and mes $(J(Q, \sigma))=0$.

Then $\chi_{s, P_{n}} \rightarrow \chi_{s, w}$ and $W_{P_{n}}(s) \rightarrow Q_{s, w}$ for any $s \in \mathbb{R}_{+}$.

Proof of Corollary 3.1 . Take $Q \in \operatorname{Per}_{1}^{*}(1)$ with $\tau(Q) \notin \mathbb{N}$. Then, there exists a sequence $P_{n} \in \mathcal{R}_{0}$ converging to $Q$. We may assume $\sigma\left(P_{n}\right) \rightarrow \sigma$ in $\mathbb{R} / \mathbb{Z}$. Since $g_{w}( \pm \sqrt{A}) \in \mathbb{C} \backslash K(Q), K(Q, \sigma)=J(Q, \sigma)$ has measure zero. Thus all the assumptions of Lemma 6.7 are fulfilled and we have $W_{P_{n}}(s) \rightarrow Q_{s, w}$. Hence the map $P \mapsto W_{P}(s)$ is discontinuous at $Q$ if $Q_{s, w} \neq Q=W_{Q}(s)$. Theorem 3.1 assures the existence of such $s$. This completes the proof.

Proposition 6.1. Each level set $\eta(Q, \sigma)=\eta$ is connected. Especially, each level set coincides with some BHL-ray.

Proof. Suppose a level set of $\eta$ consists of more than one connected components. We may assume such components lie in the strip $Q_{k}<Q<Q_{k+1}$ in $W$ for some $k \in \mathbb{N}$. In this strip, there exist only finitely many components with locally maximal or minimal levels since the map $Q \mapsto \eta(Q, \sigma)$ is real analytic for any $\sigma$. Among the components of locally maximal levels in that strip, we take a leftmost one, which we denote by $\gamma^{\prime}$. Let $\eta_{0}$ be its level. And let $\gamma^{\prime \prime}$ be the leftmost one but $\gamma^{\prime}$ among the level curves of level $\eta_{0}$; see Figure 5. By Lemma 4.1, the set $I\left(\eta_{0}\right)$ is equal to the projection of the level set $\eta(Q, \sigma)=\eta_{0}$ to the $Q$-axis. We denote the projections of $\gamma^{\prime}$ and $\gamma^{\prime \prime}$ by $\left[Q_{-}^{\prime}, Q_{+}^{\prime}\right]$ and $\left[Q_{-}^{\prime \prime}, Q_{+}^{\prime \prime}\right]$ respectively. Then, $I\left(\eta_{0}\right)$ includes $\left[Q_{-}^{\prime}, Q_{+}^{\prime}\right] \cup\left[Q_{-}^{\prime \prime}, Q_{+}^{\prime \prime}\right]$. But, because of the leftmostness of $\gamma^{\prime}$ and $\gamma^{\prime \prime}$, the region $\left\{w ; \eta(w)>\eta_{0}\right\}$ sits to the right of $\gamma^{\prime \prime}$. Hence $I(\eta)$ for any $\eta>\eta_{0}$ lies in $Q>Q_{-}^{\prime \prime}$. Since $Q_{-}^{\prime}<Q_{-}^{\prime \prime}$ by Corollary 6.2, this implies $I(\eta)$ is discontinuous at $\eta=\eta_{0}$. This contradicts Proposition 5.1. 


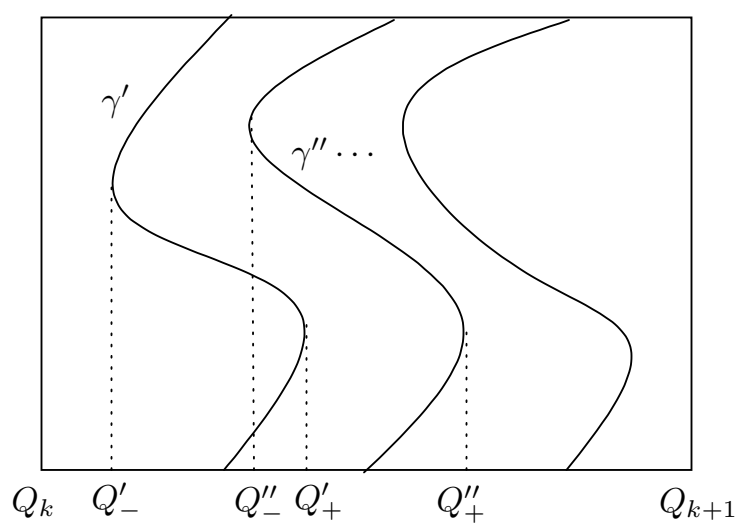

Figure 5. The level set of $\eta$ of level $\eta_{0}$

Proof of Theorem 4.1. From Lemma 4.1] $I(\eta)$ is the projection to the $Q$-axis of the set $\eta(Q, \sigma)=\eta$ in $W$. By Proposition 6.1, this level set of $\eta$ is nothing but a BHL-ray. This completes the proof.

Under the situation in Lemma 6.7, we also have the following.

Lemma 6.8. Under the same assumptions as in Lemma 6.7, we have $\eta\left(P_{n}\right) \rightarrow$ $\eta(Q, \sigma)$ and $\sigma\left(P_{n, s}\right) \rightarrow \Sigma(s, w)$ in $\mathbb{R} / \mathbb{Z}$.

Proof. Under the situation in Lemma 6.7, take a sequence $N_{n} \in \mathbb{N}$ so that $\sigma\left(P_{n}\right)+$ $N_{n} \rightarrow \sigma$. Then,

$$
\begin{aligned}
P_{n}^{N_{n}} & =\psi_{P_{n},+} \circ T_{\sigma\left(P_{n}\right)} \circ \phi_{P_{n},-} \circ P_{n}^{N_{n}} \\
& =\psi_{P_{n},+} \circ T_{\sigma\left(P_{n}\right)+N_{n}} \circ \phi_{P_{n},-} \\
& \rightarrow \psi_{Q,+} \circ T_{\sigma} \circ \phi_{Q,-} \\
& =g_{Q, \sigma}, \\
\eta\left(P_{n}\right) & =\frac{1}{\log 3}\left(\log \log \varphi_{P_{n}} \circ P_{n}^{N_{n}}\left(-\sqrt{A_{n}}\right)-\log \log \varphi_{P_{n}} \circ P_{n}^{N_{n}}\left(\sqrt{A_{n}}\right)\right) \\
& \rightarrow \frac{1}{\log 3}\left(\log \log \varphi_{Q} \circ g_{Q, \sigma}(-\sqrt{A})-\log \log \varphi_{Q} \circ g_{Q, \sigma}(\sqrt{A})\right) \\
& =\eta(Q, \sigma) .
\end{aligned}
$$

Hence, we also have

$$
\begin{aligned}
P_{n, s}^{N_{n}} & =\chi_{s, P_{n}} \circ P_{n}^{N_{n}} \circ \chi_{s, P_{n}}^{-1} \\
& \rightarrow \chi_{s, w} \circ g_{w} \circ \chi_{s, w}^{-1} \\
& =g_{Q_{s, w}, \Sigma(s, w)} .
\end{aligned}
$$

On the other hand, since

$$
\begin{aligned}
\phi_{P_{n, s},+} \circ P_{n, s}^{N_{n}}\left(\sqrt{A\left(P_{n, s}\right)}\right) & =T_{\sigma\left(P_{n, s}\right)} \circ \phi_{P_{n, s},-} \circ P_{n, s}^{N_{n}}\left(\sqrt{A\left(P_{n, s}\right)}\right) \\
& =T_{\sigma\left(P_{n, s}\right)+N_{n}} \circ \phi_{P_{n, s},-}\left(\sqrt{A\left(P_{n, s}\right)}\right) \\
& =\sigma\left(P_{n, s}\right)+N_{n},
\end{aligned}
$$


it follows that

$$
\sigma\left(P_{n, s}\right)+N_{n} \rightarrow \phi_{Q_{s, w},+} \circ g_{Q_{s, w}, \Sigma(s, w)}\left(\sqrt{A_{s, w}}\right)=\Sigma(s, w) .
$$

This implies $\sigma\left(P_{n, s}\right) \rightarrow \Sigma(s, w)$ in $\mathbb{R} / \mathbb{Z}$.

So, roughly speaking, if $P \in \mathcal{R}_{0}$ is close to $Q \in \operatorname{Per}_{1}^{*}(1)$ and $\sigma(P)$ is close to $\sigma$ in $\mathbb{R} / \mathbb{Z}$, then $\eta(P)$ is close to $\eta(Q, \sigma)$. This suggests that each S-ray in $\mathcal{R}_{0}$ can be asymptotically approximated by some BHL-ray. Since $\eta(Q, \sigma+1)=\eta(Q, \sigma)$, the BHL-rays have natural oscillation with respect to $\sigma$. This lemma suggests that, after rescaling, the oscillation of the S-ray $R(\eta)$ is similar to that of the BHL-ray $\eta(Q, \sigma)=\eta$.

Consider the $\sigma$-impression of an S-ray $R(\eta)$ :

$$
I_{\eta}(\sigma):=\left\{Q \in \operatorname{Per}_{1}^{*}(1) ; \exists P_{n} \in R(\eta) \rightarrow Q, \sigma\left(P_{n}\right) \rightarrow \sigma \text { in } \mathbb{R} / \mathbb{Z}\right\} .
$$

Proposition 6.2 ([KN, Theorem 7.1]). $I_{\eta}(\sigma)=\left\{Q \in \operatorname{Per}_{1}^{*}(1) ; \eta(Q, \sigma)=\eta\right\}$.

It is easy to see that $I(\eta)=\bigcup_{\sigma \in \mathbb{R} / \mathbb{Z}} I_{\eta}(\sigma)$.

It is expected that the $\sigma$-impression $I_{\eta}(\sigma)$ of $R(\eta)$ consists of a single point and depends continuously on $(\eta, \sigma)$. The next lemma follows from Propositions 6.2 and 6.1 .

Lemma 6.9. For a fixed $\eta_{0}$, the set $I_{\eta_{0}}\left(\sigma_{0}\right)$ consists of a single point for any $\sigma_{0}$ if and only if the BHL-ray of level $\eta_{0}$ is parametrized by $\sigma$. If the BHL-ray passes through a point $w_{0}:=\left(Q^{(0)}, \sigma_{0}\right)$, this is equivalent to the fact that the map $s \mapsto \Sigma\left(s, w_{0}\right)$ is injective.

Figure 6 describes a situation where the map $s \mapsto \Sigma\left(s, w_{0}\right)$ is not injective.

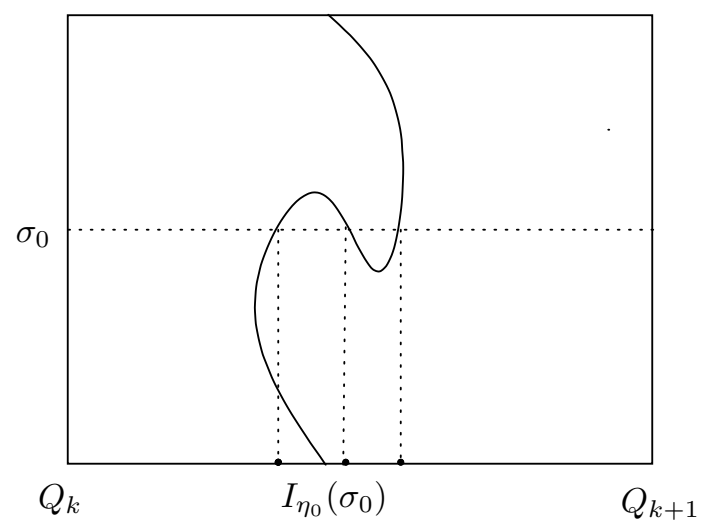

Figure 6. A $\sigma$-impression that consists of three points

Question 6.1. Is the map $s \mapsto \Sigma(s, w)$ injective as a map $\mathbb{R}_{+} \rightarrow \mathbb{R}$ ?

We will give a partial answer to this question. Recall that $Q_{k} \in \operatorname{Per}_{1}^{*}(1)$ is the map with Fatou vector $k$ for $k \in \mathbb{N}$. Then the line $Q=Q_{k}$ in $W$ forms a BHL-ray. Any BHL-ray passing through $(Q, \sigma)$ with $Q_{k}<Q<Q_{k+1}$ stays in this strip.

Lemma 6.10. If $Q \in \operatorname{Per}_{1}^{*}(1)$ is close to the map $Q_{k} \in P e r_{1}^{*}(1)$ for some $k$, then the map $s \mapsto \Sigma(s, w)$ is strictly increasing, hence injective for any $\sigma$. 
Proof. By Lemma 6.5 we have only to show the injectivity on the interval $1 \leq s \leq 3$. From the proof of Lemma 3.3, it follows that

$$
\Sigma(s, w)=\phi_{Q_{s, w},+} \circ \varphi_{Q_{s, w}}^{-1} \circ \ell_{s} \circ \varphi_{Q} \circ \psi_{Q,+}(\sigma) .
$$

Especially if $Q=Q_{k}$, then $Q_{s, w} \equiv Q$. Hence

$$
\Sigma(s, w)=\phi_{Q,+} \circ \varphi_{Q}^{-1} \circ \ell_{s} \circ \varphi_{Q} \circ \psi_{Q,+}(\sigma),
$$

and we have $\partial \Sigma(s, w) / \partial s>0$. If $Q$ is close to $Q_{k}$, then by continuity, the same inequality holds and the map $s \mapsto \Sigma(s, w)$ is injective.

For general $Q$, since $Q_{s, w}$ depends on $s$, it is difficult to estimate $\partial \Sigma(s, w) / \partial s$.

\section{ACKNOWLEDGMENT}

The author would like to thank the referee for helpful comments and advice.

\section{REFERENCES}

[B] B. Branner: Turning around the connectedness locus. In: "Topological methods in modern Mathematics." pp. 391-427. Houston, Publish or Perish, 1993. MR.1215972 (94c:58168)

$[\mathrm{BH}]$ B. Branner and J. Hubbard: The iteration of cubic polynomials. Part I: The global topology of parameter space. Acta Math. 160 (1988), pp. 143-206. MR945011 (90d:30073)

[D] A. Douady: Conjectures about the Branner-Hubbard motion of Cantor sets in $\mathbb{C}$. In: "Dynamics on the Riemann sphere." pp. 209-222. Euro. Math. Soc., 2006. MR2348963 (2008j:37103)

[KN] Y. Komori and S. Nakane: Landing property of stretching rays for real cubic polynomials. Conformal Geometry and Dynamics 8 (2004), pp. 87-114. MR2060379 (2005d:37092)

[M] J. Milnor: Remarks on iterated cubic maps. Experimental Math. 1 (1992), pp. 5-24. MR:1181083 (94c:58096)

[PT] C. L. Petersen and Tan Lei: Branner-Hubbard motions and attracting dynamics. In: "Dynamics on the Riemann sphere." pp. 45-70. Euro. Math. Soc., 2006. MR2348954 $(2008 \mathrm{j}: 37092)$

[S] M. Shishikura: The Hausdorff dimension of the boundary of the Mandelbrot set and Julia sets. Annals Math. 147 (1998), pp. 225-267. MR1626737 (2000f:37056)

[T] Tan Lei: Stretching rays and their accumulations, following Pia Willumsen. In: "Dynamics on the Riemann sphere." pp. 183-208. Euro. Math. Soc., 2006. MR.2348962 (2008i:37094)

[W] P. Willumsen: Holomorphic dynamics: On accumulation of stretching rays. Ph.D. thesis Tech. Univ. Denmark, 1997.

Tokyo Polytechnic University, 1583 Iiyama, Atsugi, Kanagawa 243-0297, Japan

E-mail address: nakane@gen.t-kougei.ac.jp 